\title{
Evaluation and Management of Patients with Hematoma After Gynecologic and Obstetric Surgery
}

\author{
Bekir KAHVECI ${ }^{1}$, Mehmet OBUT ${ }^{1}$, Serhat EGE ${ }^{1}$, Mete SUCU², Nurullah PEKER ${ }^{3}$, Osman UZUNDERE ${ }^{4}$, \\ Gaye KAHVECI ${ }^{5}$, Mehmet Sukru BUDAK ${ }^{1}$ \\ Diyarbakir Turkey
}

\begin{abstract}
OBJECTIVE: Postoperative hematoma following abdominal surgery is relatively rare and mainly depends on the type of surgery. Specific treatment including surgery or interventional radiology is sometimes necessary. The aim of this study is to evaluate the cases of postoperative hematoma after gynecologic and obstetric surgery.
\end{abstract}

STUDY DESIGN: This is a retrospective cohort study of 30 patients with hematoma developed after gynecologic and obstetric surgery. We included the patients who hospitalized with the diagnosis of a postoperative hematoma between June 2017 and April 2019 at Gazi Yasargil Training and Research Hospital of Health Sciences University. Hematomas occurring after endoscopic surgery and episiotomy were not included. The diagnosed cases were divided into three groups as wound hematoma, rectus sheath hematoma and intra-abdominal hematoma (intraperitoneal and retroperitoneal). All cases were assessed by patient demographics and clinical findings, hematoma of characteristics, treatment methods and results.

RESULTS: A total of 30 patients were included in the study with a mean age of $33.0 \pm 8.6$ years. Incidence of hematoma account for $0.2 \%$. The mean c-reactive protein was $37.9 \pm 47.4 \mathrm{mg} / \mathrm{dL}$ at admission and $14.6 \pm 25.8 \mathrm{mg} / \mathrm{dL}$ at discharge, respectively. The decrease was statistically significant $(p<$ 0.001 ). The mean hemoglobin was $10.6 \pm 2.1 \mathrm{~g} / \mathrm{dL}$ at admission and $10.7 \pm 1.5 \mathrm{~g} / \mathrm{dL}$ at discharge. Fever was detected in $7(23.3 \%)$ patients. Only 12 patients (40\%) were followed up by observation and symptom management. In $10(33.3 \%)$ patients, antibiotics were included in the treatment due to infection. In addition, 4 patients $(13.3 \%)$ had relaparotomy, 5 patients $(16.7 \%)$ underwent percutaneous radiological drainage and $8(26.7 \%)$ received blood transfusion. The mean time of resorption of the hematoma was $4.6 \pm 2.0$ days. The evaluation of the hematoma locations revealed that 14 patients $(46.7 \%)$ had wound hematoma, 7 patients $(23.3 \%)$ had rectus sheath hematoma (Type I: 2 cases, type II: 3 cases, type III: 2 cases), 8 patients $(26.7 \%)$ had pelvic hematoma and 2 patients $(6.7 \%)$ had a retroperitoneal hematoma. The mean hematoma size was $68.1 \pm 15.18 \mathrm{~mm}$.

CONCLUSIONS: In cases of hematoma resistant to antibiotic treatment and non-resorbable hematoma, we can consider percutaneous catheter drainage as an alternative to surgical intervention.

Keywords: Gynecologic and obstetric surgery, Percutaneous catheter, Postoperative hematoma drainage.

Gynecol Obstet Reprod Med 2021;27(1):60-64

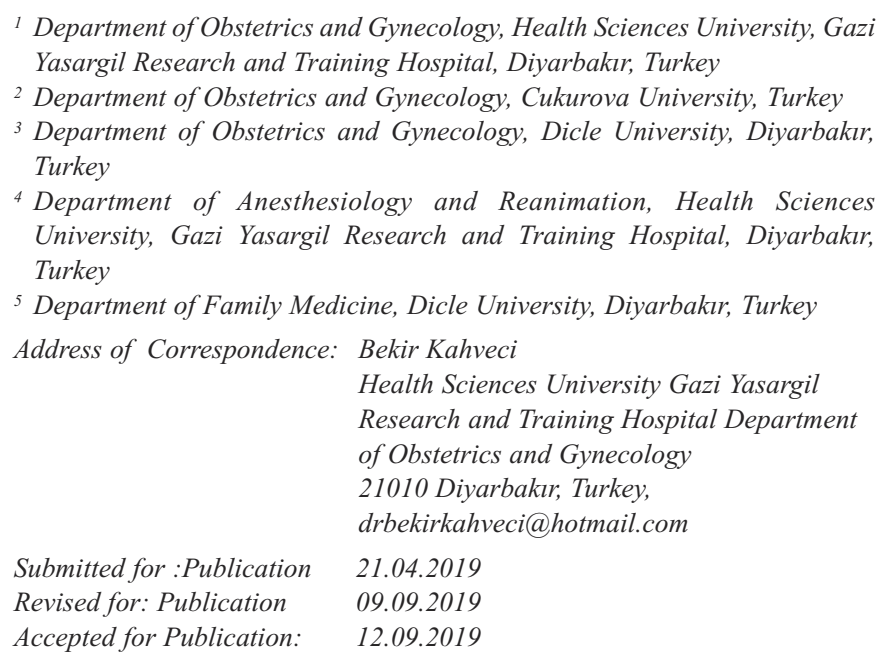

\section{Introduction}

Postoperative hematoma $(\mathrm{PH})$ after abdominal surgery (including general surgery, colorectal, orthopedic, urologic, gy-

ORCID IDs of the authors:

KB: 0000-0002-8729-1669, MO: 0000-0002-6925-4784

SE: 0000-0001-5430-602X, MS: 0000-0002-6889-7147

NP: 0000-0002-3285-9990, OU: 0000-0001-9791-0243

GK: 0000-0002-7492-0470, MSB: 0000-0001-7328-4188

\begin{tabular}{|c|c|}
\hline Quick Response Code: & Access this article online \\
\cline { 2 - 2 } & $\begin{array}{l}\text { Website: www.gorm.com.tr } \\
\text { e- mail: info@gorm.com.tr }\end{array}$ \\
\cline { 2 - 2 } & DOI:10.21613/GORM.2019.961 \\
\hline
\end{tabular}

How to cite this article: Kahveci B.Obut M. Ege S. Sucu M. Peker N. Uzundere O. Kahveci G. Budak MS. Evaluation and Management of Patients with Hematoma after Gynecologic and Obstetric Surgery Gynecol Obstet Reprod Med 2021;27(1):60-64 
necologic and obstetric procedures) is mostly asymptomatic (1), however, some patients may present with symptoms such as decreased hemoglobin levels due to the severity of infection or hematoma, which then may lead to anemia, hypovolemia symptoms, and pelvic discomfort and pain due to the mass effect associated with hematoma. It is mostly seen in patients at high risk of vascular injury due to undergoing oncologic resection $(65 \%)$, difficult anatomic exposure $(63 \%)$, previous operation (48\%), recurrent tumor (28\%) and radiation therapy (20\%) (2), however, PH may also occur in low-risk patients due to insufficient hemostasis. The localization of a hematoma may present as wound hematoma (WH), rectus sheath hematoma $(\mathrm{RSH})$ or intra-abdominal hematoma (intraperitoneal or retroperitoneal). In obstetric or gynecologic surgery, the intra-abdominal hematomas are mostly localized in Douglas, subvesical space, ischioanal fossa and broad ligament (3). They most frequently occur after abdominal and vaginal hysterectomy. PH may result in severe morbidity especially in cases of infection and significant enlargement. In cases of infected $\mathrm{PH}$, the use of prophylactic antibiotics is recommended to prevent increased morbidity (4). Ultrasound (USG), computerized tomography or relaparotomy can be used as diagnostic tools in cases of suspected PH. Today, patients safely undergo routine USG examination for the diagnosis of hematoma at the initial phase. The management of PH cases varies according to the clinical status of patients. Small, uninfected hematomas with hemodynamic stability can be followed up by expectant management, and in some cases, it is possible to perform drainage of hematoma and embolization of abnormal vessels causing hematoma by interventional radiology procedures in suitable patients (5). However, relaparotomy may be required to provide hemostasis due to hemodynamic instability caused by large hematomas. In addition, blood transfusions may be necessary for some patients and may require prolonged hospitalization.

The aim of this study was to evaluate the PH cases occurring after gynecologic and obstetric operations.

\section{Material and Method}

This cohort study was designed retrospectively after obtaining the approval of the ethics committee (2018-114) and was conducted in accordance with the Declaration of Helsinki. In the study, we included the patients who underwent abdominal surgery for obstetric or gynecologic causes and were hospitalized with the diagnosis of PH between June 2017 and April 2019 at Gazi Yasargil Training and Research Hospital of Health Sciences University. Hematomas occurring after endoscopic surgery and episiotomy were not included in the study. The patient data were obtained by examining the medical records through the hospital automation system. Ultrasound (USG) was used for the diagnosis in patients with suspected PH. The diagnosis was confirmed by aspiration with $22 \mathrm{G}$ spinal needles in patients with accessible localization of hematomas (WH, RSH and intraperitoneal). In addition, the diagnosis of hematoma was confirmed by computed tomography (CT) in cases with suspicious findings on USG or those located unfitted for needle aspiration. The diagnosed cases were divided into three groups as WH, RSH and intra-abdominal hematoma (intraperitoneal and retroperitoneal). RSH was divided into three subgroups as type I, II, III. C-reactive protein (CRP) and fever were followed up to assess the infection status in cases with PH. All cases were assessed by age, gravida, parity, abortus, hypertension, diabetes mellitus, type of operation (gynecologic or obstetric), white blood count (WBC), aPTT (activated partial thromboplastin time), INR (International normalized ratio), hemoglobin, CRP, blood transfusion requirement, treatment method (expectant followup, drainage with interventional radiology or relaparotomy), hematoma size $(\mathrm{mm})$, and the resorption time of hematomas. Antibiotics were included based on the clinical status of the patients followed up by expectant management.

\section{Statistical analysis}

IBM SPSS 20 software (IBM Corp. Released 2011. IBM SPSS Statistics for Windows, Version 20.0. Armonk, NY: IBM Corp.) was used for analyzing the data. Continuous variables were given as Mean \pm Standard deviation and median (Minimum-Maximum) values and categorical variables as $n$ $(\%)$ values. The Kolmogorov-Smirnov test was used for the assumption of normality. The paired t-test was used for the normally distributed parameters at admission and discharge, and Wilcoxon signed-rank test was used for the non-normally distributed data. The value $\mathrm{p}<0.05$ was accepted as statistically significant.

\section{Results}

During the study period, a total of 12906 patients underwent open surgery including cesarean section in 11310 patients and gynecologic surgery in 1596 patients and the incidence of hematoma was $0.2 \%$. Patient demographics and clinical findings are shown in Table I. A total of 30 patients were included in the study with a mean age of $33.0 \pm 8.6$ years (median: 33.0, range: 19-47). The mean gravida in female patients was $3.2 \pm 1.8$ (median: 3 , range: $1-6$ ), the mean parity was 2.5 \pm 1.2 (median: 2 , range: $1-5$ ) and the mean abortus was $0.7 \pm$ 0.8 (median: 0.5 , range: $0-3$ ). The evaluation of chronic diseases revealed that there were $5(16.7 \%)$ patients with DM. Similarly, there were $5(16.7 \%)$ patients with HT. The mean CRP was $37.9 \pm 47.4 \mathrm{mg} / \mathrm{dL}$ at admission, and $14.6 \pm 25.8$ $\mathrm{mg} / \mathrm{dL}$ at discharge, respectively. The decrease was statistically significant $(p<0.001)$. The mean $\mathrm{Hb}$ was $10.6 \pm 2.1 \mathrm{~g} / \mathrm{dL}$ at admission, and $10.7 \pm 1.5 \mathrm{~g} / \mathrm{dL}$ at discharge. The similarity between the $\mathrm{Hb}$ at admission and discharge was statistically significant ( $p=0.638)$. Fever was detected in $7(23.3 \%)$ patients. The mean WBC was determined as $13.5 \pm 3.8$, the mean INR as $1.2 \pm 0.3$, aPTT as $33.9 \pm 8.4$, and the platelet count as $289.7 \pm 117.1$ (count*109/L). 
Table I: Patient demographics and clinical characteristics

\begin{tabular}{ll}
\hline Parameter & Value* $^{*}$ \\
\hline Age & $33.0 \pm 8.6$ \\
Gravida & $3.2 \pm 1.8$ \\
Parity & $2.5 \pm 1.2$ \\
Abortus & $0.7 \pm 0.8$ \\
HT & $5(16.7)$ \\
DM & $5(16.7)$ \\
CRP mg/dl admission & $37.9 \pm 47.4$ \\
CRP mg/dl discharge & $14.6 \pm 25.8$ \\
Hb g/dl admission & $10.6 \pm 2.1$ \\
Hb g/dl discharge & $10.7 \pm 1.5$ \\
Fever & $7(23.3)$ \\
WBC & $13.5 \pm 3.8$ \\
INR & $1.2 \pm 0.3$ \\
aPTT & $33.9 \pm 8.4$ \\
Platelet count, *109/L & $289.7 \pm 117.1$ \\
\hline
\end{tabular}

aPTT: Activated partial thromboplastin time, INR: International normalized ratio, CRP: C-reactive protein, Hb: Hemoglobin, WBC: White blood count, HT: Hypertension, DM: Diabetes mellitus, *No of patients (\%) or mean $\pm S D$.

Treatment methods and results are shown in Table II. Only 12 patients $(40 \%)$ included in the study were followed up by observation and symptom management. In 10 (33.3\%) patients, antibiotics were included in the treatment due to infection. In addition, 4 patients $(13.3 \%)$ had relaparotomy, 5 patients $(16.7 \%)$ underwent percutaneous radiological drainage and $8(26.7 \%)$ received blood transfusion. The mean time of resorption of the hematoma was $4.6 \pm 2.0$ days.

Table II: Treatment and outcomes

\begin{tabular}{ll}
\hline Parameter & Value $^{*}$ \\
\hline Observation and symptom management alone & $12(40)$ \\
Antibiotic treatment & $10(33.3)$ \\
Blood transfusion & $8(26.7)$ \\
Percutaneous radiological drainage treatment & $5(16.7)$ \\
Relaparotomy & $4(13.3)$ \\
Time to hematoma resorbed (day) & $4.6 \pm 2.0$ \\
\hline
\end{tabular}

${ }^{*}$ No. of patients (\%) or mean $\pm S D$.

The hematoma characteristics are shown in Table III. The evaluation of the hematoma locations revealed that 14 patients (46.7\%) had WH, 7 patients (23.3\%) had RSH (Type I hematoma: 2 cases, type II hematoma: 3 cases, type III hematoma: 2 cases), 8 patients $(26.7 \%)$ had pelvic hematoma and 2 patients $(6.7 \%)$ had a retroperitoneal hematoma. The most common postoperative hematoma was WH. The distribution of hematomas is shown graphically in Figure 1. Depending on the surgical method, hematoma occurred in 18 patients $(60 \%)$ after cesarean section and in 12 patients $(\% 40)$ after gynecologic surgery. The mean hematoma size was 68.1 $\pm 15.18 \mathrm{~mm}$.
Table III: Hematoma of characteristics

\begin{tabular}{|c|c|}
\hline Location and size & Value* \\
\hline WH & $14(46.7)$ \\
\hline RSH & $7(23.3)$ \\
\hline Type I & $2(6.6)$ \\
\hline Type II & $3(10)$ \\
\hline Type III & $2(6.6)$ \\
\hline Intraperitoneal hematoma (Pelvic) & $8(26.7)$ \\
\hline Retroperitoneal hematoma & $2(6.7)$ \\
\hline Hematoma size (mm) & $68.1 \pm 15.18$ \\
\hline After Caesarean & $18(60.0)$ \\
\hline After gynecologic surgery & $12(40.0)$ \\
\hline
\end{tabular}

WH: Wound hematoma, RSH: Rectus sheath hematoma. ${ }^{*}$ No. of patients $(\%)$ or mean $\pm S D$.

Figure 1: Distribution of hematomas

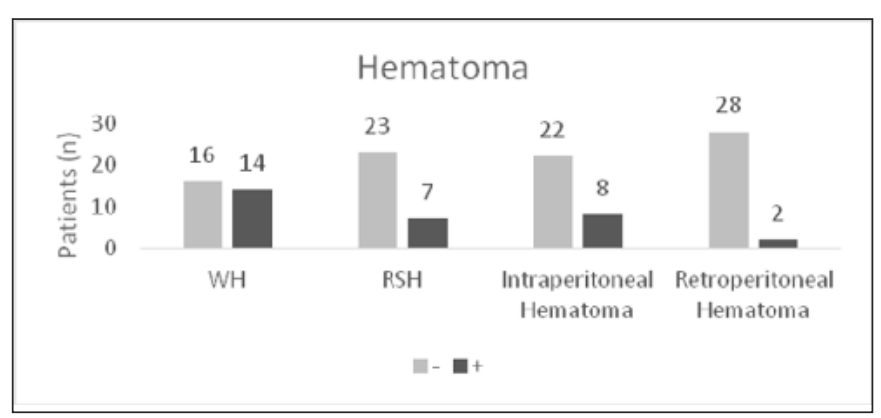

WH: Wound hematoma, RSH: Rectus sheath hematoma

\section{Discussion}

Formation of a hematoma caused by postoperative hematoma is a nightmare for all surgeons. Therefore, it is of great importance to perform a hematological assessment of patients to determine bleeding risk prior to surgery. The bleeding may be hematological or as a result of surgical procedures. It is important to distinguish between them.

In general, there is no increased bleeding tendency in elective gynecologic operations, however, the risk comes to the fore especially in surgical procedures for gynecologic cancer. A retrospective study of 351 cases revealed that PT and aPTT were prolonged in 12 patients $(3.4 \%)$ and that fibrinogen and d-dimer levels were high in $57(18 \%)$ patients (6). In our study, we determined the mean INR as $1.2 \pm 0.3$, aPTT as 33.9 \pm 8.4 , and platelet count as $289.7 \pm 117.1$ (count*109/L) within the normal reference range.

Both CT and ultrasonography were determined as effective imaging methods in the diagnosis of hematoma (7). Although USG is inadequate in the diagnosis of the hematoma resulting from bleeding in solid organs, it is an effective method to diagnose hematoma caused by intra-abdominal bleeding (8). USG appears to be a frequently preferred method for the diagnosis of postoperative hematoma as it is an accessible, non-invasive and accurate diagnostic tool. Therefore, 
USG is routinely used for the diagnosis of hematoma at the initial phase without causing harm to patients. USG also appears to be preferred for guiding hematoma drainage. CT is especially useful in the abdomen and effective in making an accurate diagnosis, which is advantageous in directing patients to the interventional radiology (9). In our study, ultrasonography was routinely used for the diagnosis of hematoma, and the expansion of hematoma in patients with planned drainage was determined by computed tomography.

A limited number of reports on pelvic hematomas after vaginal hysterectomy reveal a wide range of incidence between 19-98\% (10). Postoperative pelvic hematoma may cause pain, fever, vaginal discharge, diarrhea and mild abdominal distention, and even paralytic ileus in larger cases. The frequency of small hematomas is much higher than large ones, however, the latter is associated with higher rates of febrile morbidity (11). In most studies, fever is frequently seen in female patients with pelvic hematoma and inflammation plays an important role in the pathophysiology of postoperative pelvic hematomas (12). In our study, fever was detected in $7(23.3 \%)$ patients and antibiotic treatment was included in the treatment of these patients. Therefore, the presence of infection should be taken into account in hematomas.

Kansaria et al. reported that vaginal drainage was required in $25 \%$ of $8 \%$ of the patients with pelvic hematoma larger than $4 \mathrm{~cm}$ (13). In our study, we determined that $16.7 \%$ of the patients underwent percutaneous drainage. Thompson et al. detected pelvic hematoma in $25 \%$ of the patients after vaginal hysterectomy, which revealed a significant correlation with postoperative morbidity including hemorrhage, anemia and recurrent hospitalization (10).

Rectus sheath hematoma is a rare clinical condition, which was first accurately described by Hippocrates and mentioned by Gallen. The most common clinical manifestations of RSH are acute abdominal pain and a palpable mass. In addition, intraperitoneal ecchymosis (Cullen's sign) shows intraperitoneal rupture and the umbilical ecchymosis (Grey Turner sign) indicates the extraperitoneal spread of the hematoma. There are several risk factors associated with RSH such as old age, anticoagulation, arteriosclerosis, hypertension, coughing, pregnancy, renal disease, intense rectus muscle contractions, abdominal surgery, and abdominal wall injections (14). Females are more prone to the development of $\mathrm{RSH}$, which is mostly caused by pregnancy (15). In rare cases, as in one of our cases, patients with RSH may present with typical findings of acute abdomen, thus causing a diagnostic dilemma in the initial examination (16). Therefore it is important to make a differential diagnosis to determine the treatment process. In our study, we determined the rate of RSH as $23.3 \%$ among the hematomas.

In 1999, Berna et al. proposed a diagnostic classification based on CT findings in patients with RSH. Type I hematomas are mild and do not require hospitalization while Type II hematomas are moderate and require hospitalization, type III hematomas are more severe, mostly affect patients receiving anticoagulants and require blood transfusions (17). In our study, 2 of 7 RSH cases had type I hematoma, 3 cases had type II hematoma and 2 cases had type III hematoma. The cases with type III received blood transfusion.

Conservative treatment is the most frequently used treatment method because hematoma is a self-limiting condition in most cases. However, conservative treatment can only opt given that hematoma does not expand and the diagnosis is accurate (18). Other treatments include complete bed rest, analgesia, ice pack application, compression, discontinuation of anticoagulants, blood transfusion if needed, correction of coagulopathy if any, and antibiotic treatment in cases with signs of infection. Non-surgical treatment should be considered inappropriate cases, but it leads to higher analgesic requirements. Surgical intervention is necessary for patients with large hematomas and hemodynamic instability. Early diagnosis allows conservative treatment even in large hematomas (19). Large hematomas may take a long time to recover, and they may also get infected. In addition, large infected hematomas are less accessible to systemic antibiotic therapy and may, therefore, require surgical or percutaneous catheter drainage. In a study carried out by Sheth et al., RSH was followed up by observation strategy in $44(38 \%)$ patients and treated by medical interventions in 57 (49.57\%) patients (pain management, transfusion and coagulopathy reversal, emergency team response and ICU), and surgical interventions in $14(12.2 \%)$ patients $(20)$. In our study, only 12 of the patients (40\%) were followed up by observation and symptom management. In 10 (33.3\%) patients, antibiotics were included in the treatment due to infection. In addition, 4 patients $(13.3 \%)$ underwent relaparotomy, 5 patients $(16.7 \%)$ underwent radiological percutaneous drainage and 8 patients received blood transfusion $(26.7 \%)$.

In conclusion, a postoperative hematoma is a rare but potentially severe complication with high morbidity. Although at presentation hematoma can appear to be a life-threatening abdominal process, the outcomes are generally good with conservative measures. Both $\mathrm{CT}$ and ultrasonography have been described as effective imaging methods to establish a diagnosis of hematoma. Conservative treatment can be applied in most cases, but if infection occurs, antibiotics should be added. Early diagnosis is essential in order to avoid morbidity or unnecessary surgery. Transabdominal procedures guided by imaging methods have become an effective and safe treatment method due to advances in drainage catheter design and interventional techniques. We can consider percutaneous catheter drainage as an alternative to surgical intervention in cases of resistant to antibiotic treatment and non-resorbable hematoma. 
Conflict of interest statement: The authors declare that they have no conflicts of interest.

\section{Funding: None}

Acknowledgments: We would like to thank Selen Begum Uzun for her assistance with the statistics used in this report.

Ethics: The study was approved by the Ethics Committee of University of Health Sciences Diyarbakır Gazi Yaşargil Education and Research Hospital in Turkey (approval number: 2018-114)

Author's contribution: BK: Writing, surgical and medical practice, design, data collection, analysis or interpretation, literature search, MO: Data collection, SE: Data collection, MS: Literature search, NP: Data collection, OU: Data collection, GK: Design, analysis or interpretation, MSB: Writing, literature search

\section{References}

1. Batish A. Sathiyathasan S. Jeyanthan K. Vault haematoma after vaginal hysterectomy. NJOG. 2014;18(2):70-2. DOI: doi: 10.3126/njog.v9i2.11768.

2. Oderich GS. Panneton JM. Hofer J. Bower TC. Cherry KJ Jr. Sullivan T. et al. Iatrogenic operative injuries of abdominal and pelvic veins: a potentially lethal complication. J Vasc Surg. 2004;39(5):931-6. doi:10.1016/ j.jvs.2003.11.040.

3. Kulkarni S. Vijaya N. Detection of vault haematoma by ultrasound scan following hysterectomy and its correlation with morbidity. JOGI. 2006;56(6):507-10.

4. Dicker RC. Greenspan JR. Strauss LT. Cowart MR. Scally MJ. Peterson HB. et al. Complications of abdominal and vaginal hysterectomy among women of reproductive age in the United States. The collaborative review of sterilization. Am J Obstet Gynecol. 1982;144(7):841-8. doi: 10.1016/0002-9378(82)90362-3.

5. Li TF. Duan XH. Li Z. Ren JZ. Zhang K. Huang GH. et al. Endovascular embolization for managing anastomotic bleeding after stapled digestive tract anastomosis. Acta Radiol. 2015;56(11):1368-72. doi:10.1177/0284185 114556492 .

6. Myers ER. Clarke-Pearson DL. Olt GJ. Soper JT. Berchuck A. Preoperative coagulation testing on a gynecologic oncology service. Obstet Gynecol. 1994;83(3): 438-44.

7. Cherry WB. Mueller PS. Rectus sheath hematoma: review of 126 cases at a single institution. Medicine (Baltimore). 2006;85(2):105-10. doi:10.1097/01.md.0000216818. $13067.5 \mathrm{a}$.

8. Tasu JP. Vesselle G. Herpe G. Ferrie JC. Chan P. Boucebci S. et al. Postoperative abdominal bleeding.
Diagn Interv Imaging. 2015;96(7-8):823-31. doi: 10.1016/ j.diii.2015.03.013.

9. Wani I. Post-caesarean rectus sheath haematoma: a case report. Marmara Medical J. 2009;22(3);240-2.

10. Thomson AJ. Sproston AR. Farquharson RG. Ultrasound detection of vault haematoma following vaginal hysterectomy. Br J Obstet Gynaecol. 1998;105(2):211-5. doi: 10.1111/j.1471-0528.1998.tb10055.x.

11. Dane C. Dane B. Cetin A. Yayla M. Sonographically diagnosed vault hematomas following vaginal hysterectomy and its correlation with postoperative morbidity. Infect Dis Obstet Gynecol. 2009;2009:91708. doi: 10.1155/ $2007 / 91708$.

12. Slimani O. Alaoui F. Jayi S. Chaara H. Bouguern H. Malhouf MA. Postoperative pelvic hematoma an unusual case after surgical treatment of ectopic pregnancy. J Clin Case Rep. 2014;4(9):422. doi: 10.4172/2165-7920. 1000422 .

13. Kansaria JJ. Parulekar SV. Gupta AS. Pilankar A. Ultrasonography for evaluation of pelvic haematomas following vaginal hysterectomy. Bombay Hosp J. 2002; 44:406-5.

14. Salemis NS. Gourgiotis S. Karalis G. Diagnostic evaluation and management of patients with rectus sheath hematoma. A retrospective study. Int J Surg. 2010; 8(4): 290-3. doi: 10.1016/j.ijsu.2010.02.011.

15. Çelebi F. Balık AA. Karakaş K. Adıgüzel H. Ören D. Rektus hematomları. AÜTD. 2000;32:131-4.

16. Khan MI. Medhat O. Popescu O. Rastogi A. Thompson T. Rectus sheath haematoma presenting as acute abdomen. ANZ J Surg. 2005;75(6):502-3. doi: 10.1111/j.14452197.2005.03406.x.

17. Berna JD. Garcia-Medina V. Guirao J. Garcia-Medina J. Rectus sheath hematoma: diagnostic classification by CT. Abdom Imaging. 1996;21(1):62-4. doi:10.1007/s002619 900011.

18. Berna JD. Zuazu I. Madrigal M. Garcia-Medina V. Fernandez C. Guirado F. Conservative treatment of large rectus sheath hematoma in patients undergoing anticoagulant therapy. Abdom Imaging. 2000;25(3):230-4. doi: $10.1007 / \mathrm{s} 002619900011$.

19. Povar M. Lasala M. Ruiz A. Povar BJ. Rectus sheath haematoma: experience in our centre. An Sist Sanit Navar. 2017;40(3):361-9. doi: 10.23938/ASSN.0044.

20. Sheth HS. Kumar R. DiNella J. Janov C. Kaldas H. Smith RE. Evaluation of risk factors for rectus sheath hematoma. Clin Appl Thromb Hemost. 2016;22(3):292-6. doi: 10.1177/1076029614553024. 